\title{
Methods of Interaction Field Extension for Precision Highspeed Femtosecond Laser Processing of Transparent Materials
}

\section{DANIIL GANIN, KONSTANTIN LAPSHIN, and SERGEY VARTAPETOV}

Physical Instrumentation Center of the A.M. Prokhorov General Physics Institute of Russian Academy of Sciences, 108840, Moscow, Troitsk, Russia

\section{Abstract}

The results of experiments on the study of methods of the focal volume elongation in the direction of the propagation of femtosecond laser pulses are given. The lengthening of the focal volume due to linear effects - interface spherical aberration, beam self-diffraction, and nonlinear effects - Kerr self-focusing is demonstrated. The

Corresponding Author: DANIIL GANIN

ganin@optosystems.ru

Received: 28 January 2018

Accepted: 15 March 2018

Published: 25 April 2018

Publishing services provided by Knowledge E

(c) DANIIL GANIN et al. This article is distributed under the terms of the Creative Commons Attribution License, which permits unrestricted use and redistribution provided that the original author and source are credited.

Selection and Peer-review under the responsibility of the PhIO Conference Committee. conditions and reasons for the formation of extended filamentary micromodifications with diameters about $2 \mu \mathrm{m}$ and a length of more than $100 \mu \mathrm{m}$ in polycarbonate samples using different methods of extending the interaction region are determined.

Keywords: femtosecond laser, microstructures, microprocessing, photodegradation, filamentation.

\section{Introduction}

Ultrafast lasers are used in various fields of science, engineering and medicine. Waveguides, optoelectronic systems, microchannels, laboratory on a chip are successfully created with the help of femtosecond (fs) lasers, and technological operations such as precision laser cutting, scribing, drilling and some others are carried out using powerful picosecond lasers. In addition, ultrafast lasers are an integral part of modern ophthalmology, for example, in corneal curvature correction [1] and cataract surgery $[2,3]$. High radiation intensity, as well as the universality of radiation absorption processes in transparent materials, are the reasons for the versatile application of ultrashort laser radiation. Ultrafast lasers can successfully perform micro-and nano-processing of polymers, glasses, fused quartz, as well as crystals, transparent ceramics and some other materials. 
The advantage of using ultrafast lasers in these applications is the almost complete absence of thermal damage in the vicinity of the machined regions $[4,5]$. The reason for this is the nonlinear radiation absorption process, which is possible due to the ultrashort pulse duration. Therefore, the optical excitation of the electronic subsystem is terminated before the electron energy is transferred to the lattice.

Considerable attention has been paid to the investigation of the micromodifications formation, which are elongated in the direction of fs laser pulses propagation. The possibility of controlled formation of elongated micromodifications is promising tool for high-speed cutting and drilling of transparent dielectrics. Currently, several techniques are used to form elongated microcavities (Figure 1), for example, the use of Bessel beams [6], Kerr filamentation [7], interface spherical aberration (ISA) [8], diffraction effects [9].

The aim of the work is an experimental comparison of the methods for interaction region elongation of single fs laser pulses with the volume of transparent materials.

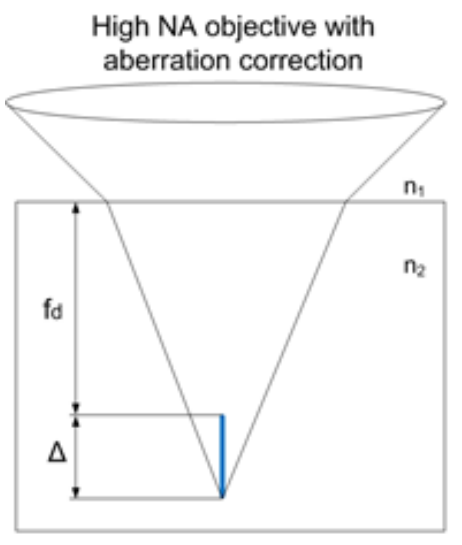

Elongation of focal volume by ISA
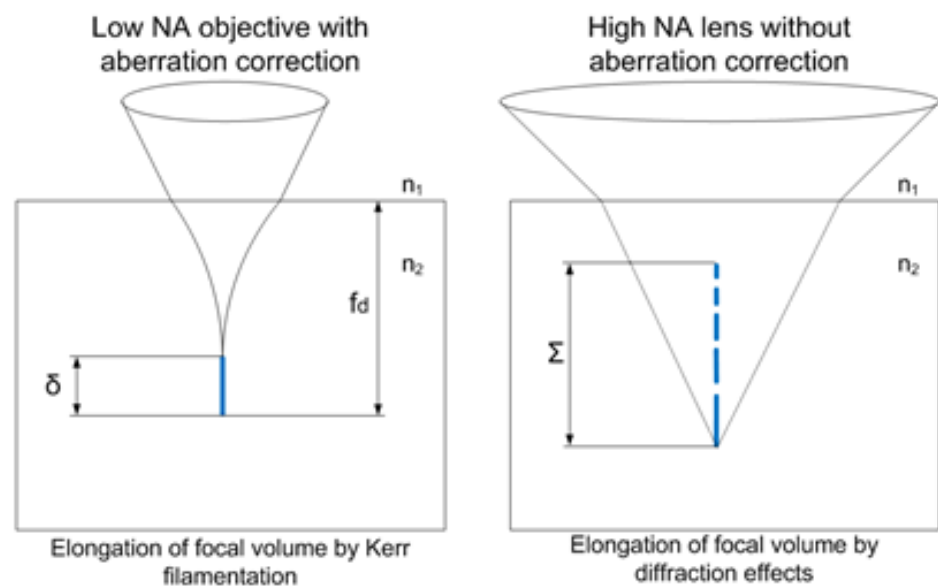

Figure 1: Scheme of formation of elongated micromodifications using different methods of lengthening the focal volume.

\section{Materials and Methods}

Fig. 2 shows the scheme of the experimental setup. We used a femtosecond regenerative Yb laser (Avesta Project, RYF-10/35) to generate femtosecond pulses at a wavelength of $1025 \mathrm{~nm}$, with pulse duration of $350 \mathrm{fs}$, pulse energy up to $150 \mu \mathrm{J}$ and repetition rate of $2000 \mathrm{~Hz}$. Pulse picker based on the Pockels cell is built into the laser.

We used the half-wave plate and the polarizer to control the energy. If necessary, the laser beam could be expanded by the LINOS (VIS-YAG $4 \mathrm{X}$ ) beam expander. We used the following focusing systems: the Leitz Wetzlar objective, PLAN L50x, NA = 0.6; 


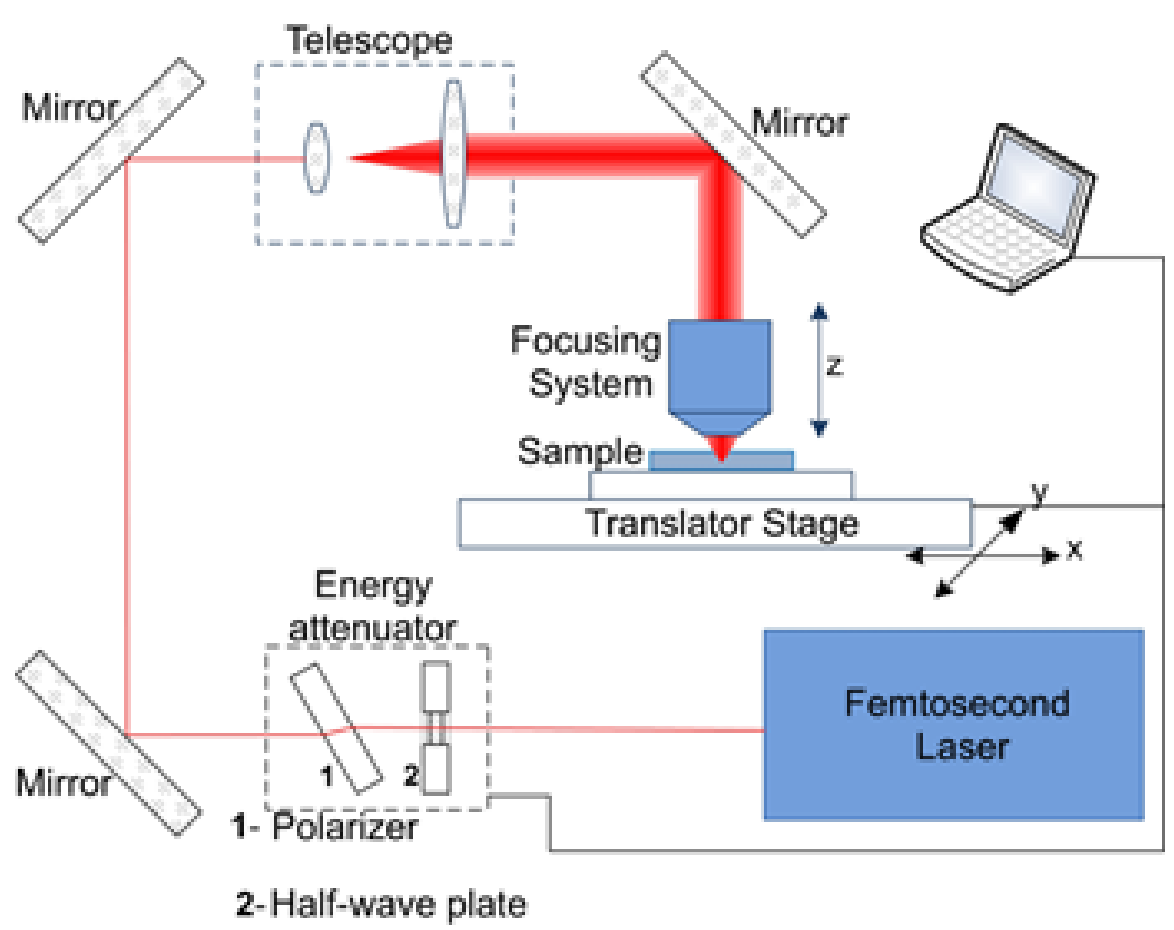

Figure 2: Experimental setup.

Lomo $\times 10$ objective NA $=0.1$ and a high aberrated spherical lens with NA $=0.58$. The lens was mounted on the z-translator, to control the focusing depth the radiation in the sample. The laser beam focused into a sample mounted on the $x$-y translator (Standa, $8 M T 173-50,1 \div 800 \mu \mathrm{m} / \mathrm{s}$ and Newport, UTS50SS, $1 \div 40000 \mu \mathrm{m} / \mathrm{s})$. The entrance surface of the sample was perpendicular to the beam. The average laser power was measured by a PD300-1W (350-1100 nm) sensor and a Nova meter (Ophir Photonics).

Polycarbonate plates (PC) with dimensions $3 \times 20 \times 50 \mathrm{~mm}$ were used as samples. The results of the action of focused fs pulses on the sample were recorded with a NIKON LV100D microscope.

The following experiment was carried out, to determine the differences in the spatial and structural modifications of the PCs formed in the samples using different focusing systems. Femtosecond laser pulses with a repetition rate of $100 \mathrm{~Hz}$ were focused through the entrance surface to the lower surface of the sample, then the sample moved along the $X$ coordinate $(V x=565.7 \mu \mathrm{m} / \mathrm{sec}$ ) and the lens moved to the top ( $\mathrm{Vz}$ $=565.7 \mu \mathrm{m} / \mathrm{sec}$ ) which led to a shift of the focal region to the entrance surface. The use of this technique made it possible to observe the structural changes created per one FS impulse, depending on the focusing depth. In other experiments, the sample was processed at a constant focusing depth. 


\section{Results}
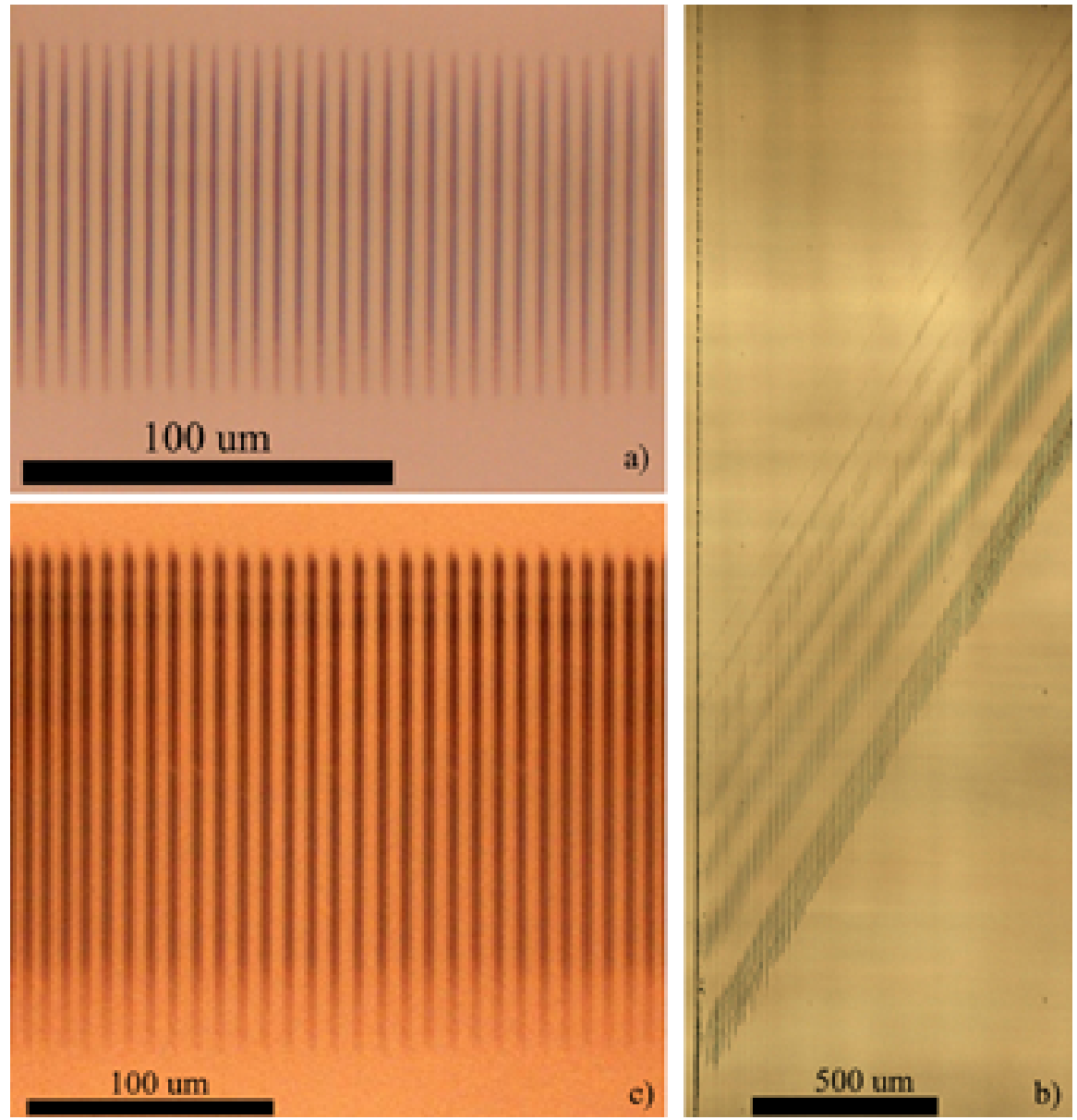

Figure 3: Photographs of linear microstructures obtained by polycarbonate micromachining with single femtosecond pulses. a) $N A=0.1, E_{\text {pulse }}=10 \mu, F=100 \mathrm{~Hz}$, focusing depth $1 \mathrm{~mm}$; b) Spherical lens, $N A=$ $\left.0.58, \mathrm{E}_{\text {pulse }}=60 \mathrm{~J}, \mathrm{~F}=100 \mathrm{~Hz} ; \mathrm{c}\right) \mathrm{NA}=0.6, \mathrm{E}_{\text {pulse }}=8 \mathrm{~J}, \mathrm{~F}=100 \mathrm{~Hz}$, focusing depth $1 \mathrm{~mm}$.

Focusing of fs laser pulses in the bulk of polycarbonate using focusing optics with $N A=0.1$ creates the necessary conditions for self-focusing and radiation filamentation, and as a result of the formation of elongated micromodifications with a length of more than $100 \mu \mathrm{m}$ and a cross-sectional diameter about $2 \mu \mathrm{m}$. Results of the action of $\mathrm{fs}$ laser pulses with an energy of $10 \mu$ in the form of linear tracks of equal length are presented at Fig. 3 a). Laser scanning was performed only along the $X$ coordinate at a speed of $800 \mu \mathrm{m} / \mathrm{sec}$. Dependence of the length of tracks on the focusing depth is not observed.

In the case of using of spherical lens with $N A=0.58$ and strong spherical aberrations as a focusing system, the volume modification has a complex spatial structure. The corresponding picture is shown in Fig. 3 (b). The scanning was simultaneously performed on the $X$ and $Z$ coordinates at $565.7 \mu \mathrm{m} / \mathrm{sec}$. It is established that a single femtosecond 
pulse creates a linear structure in the sample along the radiation propagation axis, consisting of a sequence of filamentary micromodifications. These micromodifications are separated by regions with a modified refractive index. The total length $(\Sigma)$ of the modified region exceeds $1000 \mu \mathrm{m}$. The dependence of the length of the modified region on the focusing depth is not observed. The line in the left part of the photo corresponds to the sample standing point ( $\approx 500$ pulses). Increasing the of the laser pulse energy to $150 \mu$ J leads to association of micromodifications in one Fig. 4.

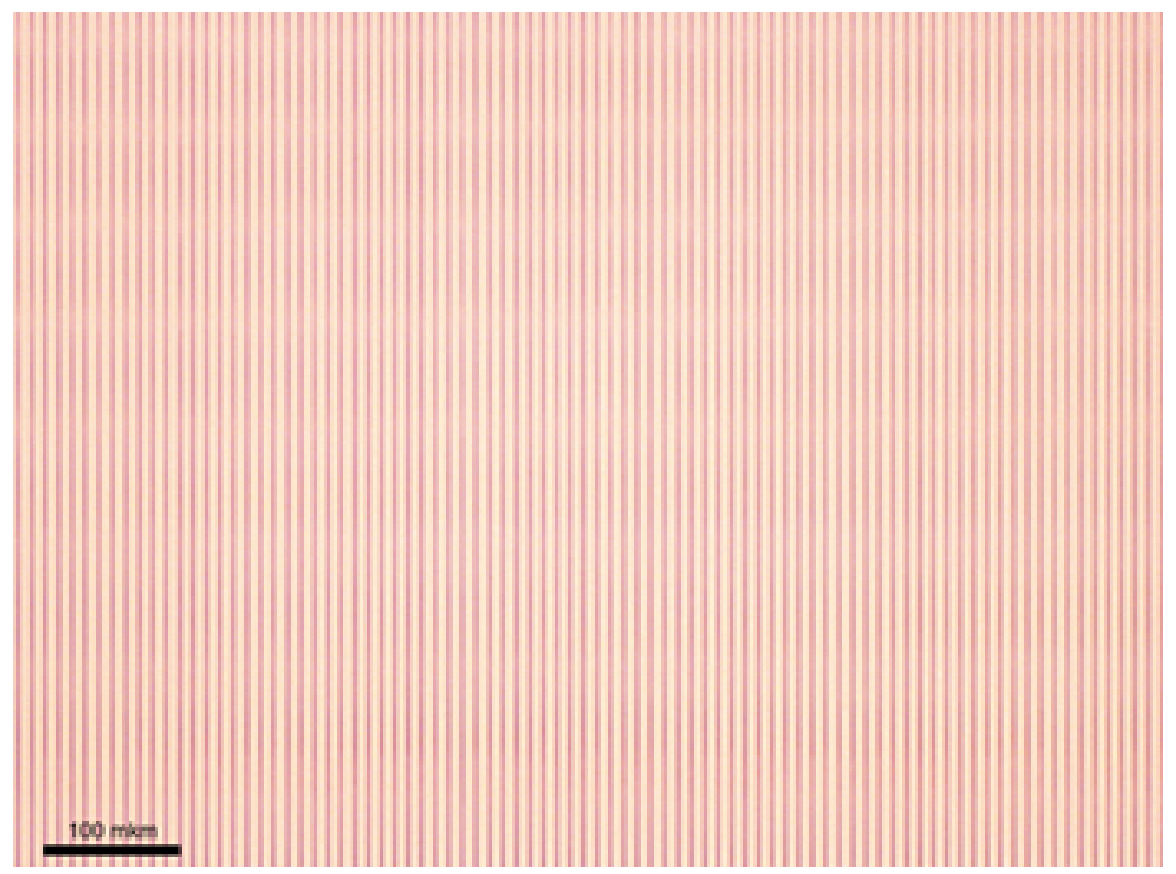

Figure 4: Photograph of filamentary micromodifications created by single fs laser pulses in the volume of $P C$ samples. The fs pulses are focused by a spherical lens with NA $=0.58$. $\mathrm{E}_{\text {pulse }}=150 \mathrm{\mu}$, the focusing depth is constant.

In the case of using objective (NA=0.6) instead of a spherical lens, whilemaintaining the other parameters of the system, FS pulse creates in a bulk only one micromodification that elongated on axis of radiation propagation (Fig. $3 \mathrm{C}$ ). In addition, it was found that increasing the focusing depth leads to an increase of the micromodification length, this corresponds to [10] and is explained by ISA at the air-sample boarder [11]. A change in the energy of the pulse leads to a decrease in the length of the filamentary micromodifications.

\section{Discussion of results}

The short duration of femtosecond pulses allows to reach the high peak powers, as well as an incredible intensity in the focal volume, which in turn can lead to nonlinear interactions with the material [12]. At the same time, it is known that the fs 
pulses focused in the sample volume can cause various modifications of the material structure: complete destruction of the material in the focal volume region, depolymerization, increase in the density of matter, graphitization and other $[13,14]$, which causes a change in the optical and electrical characteristics of the material. The nature of the modifications depends on a number of parameters: the energy of the pulse, its duration, the characteristics of the energy distribution in the cross section of the beam, the depth of focusing, the scanning speed, and the properties of the material. The propagation of pulses with a high peak power can lead to Kerr's self-focusing, and as a consequence, to the collapse of the laser beam and filamentation. The formation of a laser filament in a volume of transparent dielectrics, together with a high intensity, leads to the formation of micromodification elongated in the direction of propagation (Fig. 1). To achieve the conditions of stable filamentation of femtosecond pulses in transparent materials, it is necessary to exceed the peak power of the pulse $P$ above the critical power $\mathrm{P}_{c r}$.

It is known $[12,15]$ that in the process of self-focusing, if the current power on the time profile of the ultrashort pulse exceeds the so-called critical power $\mathrm{Pcr}$, then the laser beam begins to focus, and the position of the nonlinear focus (the focusing length) is determined by the Marburger formula:

$$
\begin{gathered}
z_{n f}=\frac{0.367 k a^{2}}{\left\{\left[\left(\frac{P_{\text {pulse }}}{P_{c r}}\right)^{1 / 2}-0.852\right]^{2}-0.0219\right\}^{1 / 2}} \\
P_{c r}=\frac{\lambda^{2}}{2 \pi n_{0} n_{2}}
\end{gathered}
$$

where $\mathrm{a}$ is the radius of the laser beam at the entrance of the medium, $\mathrm{k}$ is the wave vector, $P_{p u l s e}$ is the maximum peak input power, $P_{c r}$ is the critical self-focusing power, $\mathrm{n}_{0}$ is the linear refractive index, and $\mathrm{n}_{2}$ is the nonlinear refractive index. If we pass the laser beam through a focusing lens at the entrance surface of the medium, the focusing spot inside the medium shifts from the geometric focus towards the objective to a new position, which determined by the ratio $P_{p u l s e} / P_{c r}$. The expression that determines the distance of displacement of the focusing spot from the position of the geometric focus $f_{d}$ with respect to the Marburger formula in this case has the form:

$$
\delta=\frac{f_{d}^{2}}{f_{d}+z_{n f}} .
$$

Thus, it is clear that the main parameter affecting on the micromodification length during filamentation of the fs laser pulse is the excess of the peak pulse power over 
the critical power, which is individual for different materials. It should also be noted that self-focusing is more responsible for the formation of filamentary micromodification before of the geometric focus [15].

In the case of focusing by optical systems with high numerical apertures designed to work in parallel beams, the form of micromodifications can be very different from the sphere, and acquire the appearance of filamentary thread elongated in the direction of radiation propagation. The main reason for increasing the aspect ratio is the interface spherical lens aberration (ISA). ISA at the air-sample boundary extends the waist region towards the propagation of radiation. The length of the waist beyond the geometric focus $\left(f_{d}\right)$ is determined by the formula proposed in $[1,11]$ :

$$
\Delta=\frac{f_{d}}{n} *\left(\sqrt{\frac{n^{2}-N A^{2}}{1-N A^{2}}}-n\right),
$$

where $n=n_{2} / n_{1}=n_{2}$, with $n_{1}=1, f_{d}$ - focusing depth, NA- numerical aperture of the objective. It can be seen that $\Delta$ (the length of the focal volume) is most sensitive to a change in the focusing depth $\left(\Delta \sim \mathrm{f}_{d}\right)$, the numerical aperture NA and indirectly to the energy of the pulse $\mathrm{E}_{\text {pulse }}$ through a change in the energy at the peripheral rays in the energy distribution along the beam cross section.

The results (focusing of radiation with an objective with correction of spherical aberrations) of the increase in filament length with increasing focusing depth is in good agreement with the formula determining $\Delta$.

Using a spherical lens as a focusing system leads to the formation of structures in the bulk of the sample that are significantly different from previous cases. Sequence of filamentary micromodifications with constant diameter (Figure $3 \mathrm{~b}$ ), arranged in succession along the propagation axis of fs laser pulse (z-axis) is formed. The length of the micromodifications in this sequence decreases towards the lens. The micromodifications are combined into a single and forming a microstructure $2 \mu \mathrm{m}$ in diameter with an aspect ratio of more than 1000 (Fig. 4) with increasing pulse energy. The reason responsible for the formation of micromodifications of the presented spatial configuration is the transformation of the initial Gaussian distribution of laser radiation intensity into a set of diffraction ring of the Fresnel type. In this case, each diffraction ring has its own geometrical focus position, i.e. each diffraction ring will be focused in the air and in the material at different depths [9]. 


\section{Conclusion}

In this paper, the results of using various methods of extending the interaction region of single fs laser pulses with the volume of transparent materials were shown. The use of linear and nonlinear methods of lengthening the focal volume can become a promising tool for increasing productivity and quality in cutting and drilling of transparent materials with fs laser pulses. It is shown that the focusing of fs pulses into the bulk of polycarbonate by objective with a small numerical aperture can lead to self-focusing, and as a consequence to the formation of an extended filamentary micromodification. In this case, the length of the micromodification does not depend on the focusing depth, and is determined by the peak power of the laser pulse. In the case of high numericaql aperture lenses, the micromodification region formed by a single fs pulse in the sample volume can be very different from the sphere and have the form of thread whose length depends on the focusing depth and the numerical aperture and is determined by the interface spherical aberration. When using a spherical lens with a large numerical aperture and strong spherical aberrations as a focusing system, a sequence of micromodifications located along the optical axis is formed in the volume of the sample under the action of a single femtosecond pulse. The total length of the modified region depends on the energy of the laser pulse and can exceed $1 \mathrm{~mm}$. The dependence of the micromodification length on the focusing depth is not observed.

\section{Acknowledgements}

This work was partially supported by Foundation for Assistance to Small Innovative Enterprises inScience and Technology, program U.M.N.I.K. (10624GU/2016 No. 0023590).

\section{References}

[1] Vartapetov S.K. et al. Femtosecond lasers for microsurgery of cornea // Quantum Electron. 2012. Vol. 42, № 3. P. 262.

[2] Juhasz T. et al. Corneal refractive surgery with femtosecond lasers // IEEE J. Sel. Top. Quantum Electron. 1999. Vol. 5, № 4. P. 902-910.

[3] Grewal D.S. et al. Femtosecond laser-assisted cataract surgery-current status and future directions // Survey of Ophthalmology. 2016. Vol. 61, № 2. P. 103-131. 
[4] Schaffer C.B. et al. Micromachining bulk glass by use of femtosecond laser pulses with nanojoule energy // Opt. Lett. 2001. Vol. 26, № 2. P. 93-95.

[5] Burakov I.M. et al. Spatial distribution of refractive index variations induced in bulk fused silica by single ultrashort and short laser pulses // J. Appl. Phys. 2007. Vol. 101 , № 4 .

[6] Rapp L. et al. High speed cleaving of crystals with ultrafast Bessel beams // Opt. Express. 2017. Vol. 25, № 8. P. 9312-9317.

[7] BUTKUS S. et al. Improvement of Cut Quality in Rapid-Cutting of Glass Method via Femtosecond Laser Filamentation Improvement of Cut Quality in Rapid-Cutting of Glass Method via // J. Laser Micro / Nanoeng. 2015. Vol. 10, № 1.

[8] Ganin D. V et al. Single-pulse perforation of thin transparent dielectrics by femtosecond lasers // Appl. Phys. A. Springer Berlin Heidelberg, 2017. Vol. 123, № 378.

[9] Ganin D. V et al. Femtosecond laser fabrication of linear graphitized microstructures in a bulk of polycarbonate samples // J. Phys. Conf. Ser. 2016. Vol. 737. P. 12023.

[10] Ganin D. V et al. Specific features of direct formation of graphite-like microstructures in polycarbonate samples by single femtosecond laser pulses // Quantum Electron. 2015. Vol. 45, № 11. P. 1029-1036.

[11] Sun Q. et al. Effect of spherical aberration on the propagation of a tightly focused femtosecond laser pulse inside fused silica // J. Opt. A Pure Appl. Opt. 2005. Vol. 7. P. 655-659.

[12] Couairon A., Mysyrowicz A. Femtosecond filamentation in transparent media // Physics Reports. 2007. Vol. 441, № 2-4. P. 47-189.

[13] Schaffer C.B., Brodeur A., Mazur E. Laser-induced breakdown and damage in bulk transparent materials induced by tightly focused femtosecond laser pulses // Meas. Sci. Technol. 2001. Vol. 12, № 11. P. 1784-1794.

[14] Juodkazis S. et al. Laser-induced microexplosion confined in the bulk of a sapphire cystal: Evidence of multimegabar pressures // Phys. Rev. Lett. 2006. Vol. 96, № 16.

[15] Couairon A. et al. Filamentation and damage in fused silica induced by tightly focused femtosecond laser pulses // Phys. Rev. B - Condens. Matter Mater. Phys. 2005. Vol. 71, № 12. 